\title{
Propuestas para la Virtualización de la Enseñanza en Ciencias Jurídicas (Un Ensayo sobre la Asignatura "Teoría de las Relaciones Laborales" del Grado en Relaciones Laborales y Recursos Humanos POR la UnIVERSIDAD DE GRANADA)
}

Amparo M. Molina Martín*

RESUMEN:

La reproducción de ambientes educativos virtuales representa una de las novedades más significativas asociadas a la implantación del Espacio Europeo de Educación Superior. Ello no obstante, existen determinados procesos de enseñanza-aprendizaje que ofrecen resistencia a su utilización, como es el caso del de las Ciencias Jurídicas. Asípues, el presente estudio está dedicado a la proposición de un sistema de soportes, contenidos, objetivos, competencias, actividades y evaluaciones con el que desarrollar la planificación didáctica de una asignatura de perfil jurídico cual es la de "Teoría de las Relaciones Laborales" del Grado en Relaciones Laborales y Recursos Humanos por la Universidad de Granada.

Palabras Clave:

Enseñanza; virtualización; Ciencias Jurídica; Grado en Relaciones Laborales y Recursos Humanos.

ABSTRACT:

The reproduction of virtual educational environments represents one of the most significant novelties associated to the introduction of the European Higher Education Area. However, there are certain teaching-learning processes which offer resistance to its use,

* Profesora Ayudante Doctora. Departamento de Derecho Financiero y de la Seguridad Social. Facultad de Derecho. UniversidaddeGranada•molina@ugr.es 
as in the case of Legal Sciences. So, the present study is dedicated to the proposal of a system of resources, contents, goals, competences, activities and evaluations which will serve to develop the didactic planning of a legal profile subject as the one of "Theory of Labour Relations" of the Degree in Labour Relations and Human Resources at the University of Granada.

KEYWORDS:

Teaching; virtualization; Legal Sciences; Degree in Labour Relations and Human Resources.

\section{1.- CONTEXTUALIZACIÓN DEL ESTUDIO}

La opinión relativamente generalizada de que el proceso de enseñanzaaprendizaje exige "presencialidad" lleva un tiempo puesta a debate a consecuencia, sobre todo, de la aparición y difusión de "ambientes educativos virtuales" -también conocidos como "ambientes virtuales de aprendizaje" o "sistemas de gestión del aprendizaje", entre otros términos-. No en vano, estos nuevos espacios -tales como Moodle, WebCT o Campus- proporcionan a la comunidad educativa un entorno de posibilidades múltiples para instrumentalizar la "virtualización" de contenidos académicos (Cebrián, 2003).

Ello no obstante, existen ciertos contextos que ofrecen resistencia a la implementación de estas singulares herramientas como consecuencia, por lo general, más de prejuicios o de costumbres que de impedimentos reales. Así suele suceder, por ejemplo, en las disciplinas pertenecientes a las Ciencias Jurídicas donde, precisamente, se incardina la asignatura concernida en el presente estudio - "Teoría de las Relaciones Laborales"-. Respecto de ésta, nótese que, conforme al Plan de Estudios vigente $^{1}$, se trata de una materia Básica incardinada en el Módulo "Historia" -junto con "Historia de las Relaciones Laborales"- del Primer Curso, segundo cuatrimestre, del Grado en Relaciones Laborales y Recursos Humanos por la Universidad de Granada y que imparte profesorado adscrito al Departamento de Derecho del Trabajo y de la Seguridad Social.

Siendo tales, a grandes rasgos, los elementos que caracterizan el escenario educativo seleccionado para la realización del presente estudio, nuestro planteamiento central consiste en procurar un acercamiento positivo entre la enseñanza virtual y el Derecho, o viceversa. La renovación del proceso de enseñanza-aprendizaje resultante de la creación del Espacio Europeo de Educación Superior, unida a la convicción

1. Disponible en http://citrab.ugr.es/contenido/files/reform/Grado_RRLL_y_RRHH.pdf (consultado a 28 de noviembre de 2011). 
personal de su procedencia, aconsejan -si no requieren- la reversión del estadio en el que se vienen encontrando sus relaciones mutuas hacia un lugar de coexistencia y colaboración. En modo alguno se pretende, entonces, establecer un manual para emprender dicho proceso; nuestra intención es meramente la de compartir reflexiones acerca de distintas herramientas disponibles para afrontarlo.

Con dicho objetivo, y a modo de guía, adelantamos que el estudio que sigue se estructura en cuatro partes a lo largo de las cuales se progresa en el desarrollo de la propuesta de virtualización, partiendo de la selección de sus soportes en tanto instrumentos para la transmisión de los contenidos en coherencia con las competencias y objetivos de la enseñanza-aprendizaje de la materia seleccionada y con el complemento de un catálogo de actividades y métodos de evaluación.

\section{2.- ELEMENTOS ESPECÍFICOS PARA LA ENSEÑANZA VIRTUAL EN CIENCIAS JURÍDICAS Y PARA LA "TEORÍA DE LAS RELACIONES LABORALES"}

\section{1.- Premisas Generales en Torno a la Planificación Didáctica}

La opción por la virtualización de la enseñanza -en principio, con independencia de la disciplina concernida- lleva aparejado el seguimiento de un proceso específico de reflexión y maduración acerca de los múltiples elementos que componen el conjunto de la planificación didáctica. Se trata, además, de una actividad que suele responder a la voluntad de el/la responsable de la materia afectada, lo cual puede llegar a convertirla en un trance con efectos disuasorios o perniciosos para su evolución y consolidación.

Desde esta perspectiva, entendemos que la enseñanza virtual demanda el establecimiento de una primera organización concreta de carácter subjetivo, de manera que su ejecución efectiva resulte de las aportaciones realizadas por los distintos actores que participan del proceso educativo. Es por tal motivo por el que proponemos una separación inicial de dos actividades: el diseño y la gestión.

A resultas de ello, el planteamiento quedaría como sigue: mientras que la primera (diseño), sería desarrollada por el profesorado -a modo de "equipo"- en el marco general de la planificación didáctica, la segunda actividad (gestión) admitiría -incluso, aconsejaría- la implicación de los estudiantes. El proceso de enseñanza-aprendizaje, mediante esta habilitación de espacios de participación, adquiriría pues un carácter dinámico que coadyuvaría a salvar las dificultades apuntadas con anterioridad y tendería a hacerlo exitoso. Consecuentemente, las herramientas o soportes, actividades y evaluaciones para su desarrollo integral habrían de ir en consonancia con el ambiente académico existente o creado en ese contexto académico a partir, en nuestra opinión, de la actitud "constructiva y proactiva" de el/la profesor/a. 
La propuesta concreta que presentamos a continuación, por tanto, sugiere un conjunto de fórmulas con el que se persigue evitar la rigidez o el estatismo del proceso de enseñanza-aprendizaje. Ahora bien, no se trata tampoco de convertirlo en algo permanentemente sujeto a alteraciones. La clave se encuentra, a nuestro entender, en lograr la conciliación de los requerimientos propios de dicho proceso y el referido ambiente académico.

Con tales premisas, además, el estudio que sigue no se circunscribe al relato de instrumentos recomendables para ser desarrollados a través de una enseñanza virtual, sino que se procura hacer un análisis completo -sintetizado en una Tabla final²- de cómo resultaría toda la planificación didáctica de la asignatura seleccionada.

\section{2.- Descripción de los Soportes en Asociación con los Contenidos}

Tomando como referencia la guía docente elaborada para "Teoría de las Relaciones Laborales"3, y aun detectada su relativa falta de adecuación a un planteamiento de enseñanza virtual, se formula la siguiente combinación de soportes y contenidos:

\section{a) Audio:}

Se considera que este soporte es idóneo para transmitir con éxito los contenidos referidos a las relaciones colectivas de trabajo (léanse: sindicatos, asociaciones empresariales, negociación colectiva, huelgas, cierres patronales, etc.). No en vano, éstos tienen una presencia elevada en los medios de comunicación, máxime a partir del acontecimiento de la crisis económica. El acceso a esta información, además, es múltiple, público y gratuito, y su utilidad para hacer comprender la dinámica del conflicto socio-laboral es elevada.

Como muestra de ello, pueden consultarse las hemerotecas online (en formato podcast) de la mayoría de emisoras de radio.

\section{b) Video:}

Sin perjuicio de lo recién expuesto -pues, como los soportes en audio, también aquéllos en vídeo son adecuados a los contenidos comentados- se entiende que la visualización de ciertas películas, series, documentales o reportajes permite transmitir el alcance y significado de la dimensión individual de las relaciones de trabajo (requisitos de los prestadores de servicios, modalidades de contratación, desarrollo de la actividad laboral, entre otros elementos). Ahora bien, esta materia en particular,

2. Véase el apartado 3.-

3. Disponible en http://citrab.ugr.es/contenido/asignat/EEES/1_Teoria_RRLL.pdf (consultado a 28 de noviembre de 2011). 
dada su amplitud y complejidad, precisa el apoyo de otros soportes eminentemente documentales.

A modo de ejemplo, nos remitimos a los filmes "Germinal" (1993), "El Efecto Iguazú" (2003) y "El Método" (2005), o a emisiones disponibles en Internet de diversos programas informativos.

\section{c) Documentos:}

Qué duda cabe de que éste es el soporte prototípico para la transmisión de contenidos en el ámbito seleccionado. Ahora bien, ello no lo convierte necesariamente en la herramienta ideal independientemente del contexto educativo. Bien al contrario, se opina que su utilización, como para el resto de soportes, debe ser selectiva y proporcionada. Así, los contenidos de "Teoría de las Relaciones Laborales" que mejor pueden transmitirse por esta vía son, precisamente, aquéllos de perfil teórico (en esencia: concepto, modelos y ámbitos de manifestación de las relaciones laborales).

A tal efecto, se dispone de literatura especializada en manuales, libros y capítulos o artículos en revistas, sin descartar el recurso a páginas web de difusión científica e institucionales.

\section{d) Otros:}

Bases de datos: disponibles a través del sitio en Internet de la Biblioteca -en nuestro caso, de la Universidad de Granada, tales como "La Ley", "Tirant Online" o "Westlaw Aranzadi"- su utilización es fundamental para la transmisión de todo tipo de contenidos en tanto soporte documental, pero también para ensayar la adquisición de competencias (generales-instrumentales y específicas-procedimentales) clave para la aprehensión de la materia.

Foros: cabe su uso para abordar los contenidos del sistema de fuentes del Derecho a partir de una breve introducción en soporte documental (textos normativos).

Eventos científicos: en su caso, se puede recurrir a cursos o formación complementaria online que aborden algún contenido particular.

\section{3.- Selección de los Soportes a Partir de la Determinación de los Objetivos y las Competencias}

Como es sabido, el proceso de enseñanza-aprendizaje, singularmente en el contexto universitario, debe estar orientado a la consecución de las competencias que habilitan para el ejercicio de la actividad profesional que dota de contenido al correspondiente Plan de Estudios. Se trata, así pues, de hacer efectivo uno de los referentes e inspiraciones principales del denominado "Proceso de Bolonia", desterrando el -hasta ahora, imperante- recurso a la "impartición" de conocimientos y contenidos 
puramente teóricos. Y es que si el Espacio Europeo de Educación Superior demanda en este punto una transformación significativa de la actividad docente -"focalizar la atención de los procesos formativos en los aprendizajes de los estudiantes y más concretamente en los resultados de aprendizaje expresados en términos de competencias" (Cano, 2008)- en el ámbito de las Ciencias Jurídicas ésta se convierte en una exigencia fundamental por ser un terreno habitualmente asociado al aprendizaje memorístico en sentido estricto.

Desde esta perspectiva, la planificación docente de la organización curricular -donde se insertan los contenidos, objetivos, sistema de evaluación, etc.- ha de tomar en consideración el conjunto de necesidades y requerimientos formativos de los estudiantes tanto con vistas a su incorporación al mercado de trabajo como en relación con cada momento de su participación en la actividad universitaria. Ello supone, por tanto, proyectar efectivamente la enseñanza-aprendizaje como un proceso que ha de avanzar progresivamente y adaptarse a cada fase de la trayectoria académica de los estudiantes. Se trata, en suma, de formar profesionales cualificados y competentes sin olvidar que, al mismo tiempo y mientras tanto, son parte de una comunidad -la comunidad universitaria- y, como tales, les corresponden ciertos intereses propios en sus manifestaciones de derechos, deberes, facultades y obligaciones.

En definitiva, la proyección de la enseñanza universitaria a través de las competencias - ergo, capacidades y habilidades que los estudiantes deben dominar para realizar sus tareas u operaciones profesionales con un determinado nivel de calidad (Blanco, 2009)- supone transformar su dinámica mediante la identificación, selección y concreción de las propias de cada disciplina implicada, así como propiciar su difusión y reproducción en toda la estructura educativa, tomando en consideración tanto el perfil profesional como la situación académica de los estudiantes.

Desde esta óptica, y para la asignatura "Teoría de las Relaciones Laborales" del Grado en Relaciones Laborales y Recursos Humanos por la Universidad de Granada, se propone un esquema de asociación entre competencias y soportes vinculados a la enseñanza virtual que se entiende adecuado para alcanzar los siguientes objetivos del proceso de enseñanza-aprendizaje:

a) Introducir los planteamientos centrales del sistema de relaciones laborales;

b) iniciar a los estudiantes en el nuevo proceso de enseñanza-aprendizaje universitario; y

c) dar a conocer la organización y servicios básicos de la comunidad (Universidad, Facultad, Departamento, etc.). 
Y a tales objetivos se hacen corresponder las siguientes competencias:

1) Capacidad para seleccionar y gestionar información y documentación laboral.

2) Capacidad para comprender el papel asignado a los actores sociales.

3) Capacidad para comprender las relaciones de poder en el ámbito de las relaciones de trabajo.

4) Capacidad para interrelacionar las distintas disciplinas que configuran las relaciones laborales.

5) Capacidad de aplicar las tecnologías de la información y la comunicación en diferentes ámbitos de actuación.

Conforme a lo expuesto, y en relación asimismo con lo indicado en el apartado anterior, la enseñanza-aprendizaje de las competencias a adquirir en dicha asignatura, mediante soportes vinculados a la enseñanza virtual, se justifica sobre distintas bases.

Así, cada competencia es susceptible de ser adquirida a través de distintos medios o soportes. Ahora bien, su elección exacta tiene consecuencias en términos de rendimiento del proceso educativo. Es por ello que se opta por adjudicar varios soportes a cada competencia siempre desde el convencimiento de que se trata de aquéllos que permiten su óptima adquisición en términos cuantitativos, cualitativos y temporales -igualmente del tiempo aconsejable se deja constancia en la Tabla final-.

La elección concreta de cada soporte, por su parte, responde a motivaciones similares. De ahí que las competencias de perfil más científico-técnico (1, 2 y 4$)$ tengan asociada la tarea de "explicación", mientras que para el resto (3 y 5) no se considera necesario.

Además, existe una relativa tendencia a apoyar la adquisición de la mayoría de las competencias en soportes dinámicos vinculados a las nuevas tecnologías de la información y la comunicación, pues se considera que el actual perfil de estudiante universitario así lo admite y hasta lo recomienda. Es por ello que se dispone con frecuencia la consulta de Internet (bases de datos online para la competencia 1; vídeos para las competencias 2, 3 y 5; páginas web para la competencia 4).

Respecto del soporte de documentos, se prefiere reservarlo para la adquisición de competencias del tipo disciplinares-cognitivas (eminentemente, 1, 2 y 4) por tratarse de aquéllas en las que el proceso de enseñanza-aprendizaje resulta más exigente. De hecho, su utilización se acompaña de explicaciones a modo de profundización y refuerzo. 


\section{4.- Seguimiento del Desarrollo del Proceso de Enseñanza-Aprendizaje Virtual:} Actividades y Evaluación

Consustanciales como son a la actividad educativa los elementos de control y evaluación, su planteamiento virtual ha de contemplarlos expresamente y en concordancia con el diseño elegido. Sobre la base del propuesto para el presente estudio, y observando nuevamente la recomendación de implicar en él al alumnado, se consideran adecuadas las siguientes actividades:

a) Carpetas de aprendizaje.

b) Presentación de diapositivas.

c) Realización de exámenes teóricos y/o prácticos.

d) Entregas de ejercicios resueltos o resultados.

e) Valoración de las aportaciones en participación e intervención activas.

f) Registros de consultas.

A las que se propone hacer corresponder uno o varios de los siguientes métodos de evaluación:

a) Listas de control (competencia de el/la profesor/a y/o autoevaluación de los/ as estudiantes)

b) Escalas de valoración (competencia de los/las estudiantes-por pares)

c) Pruebas de respuestas "de desarrollo" (competencia de el/la profesor/a)

d) Pruebas objetivas (competencia de el/la profesor/a)

e) Rúbricas (competencia de el/la profesor/a)

f) Concursos-debates (competencia de el/la profesor y/o de los/as estudiantespor pares)

\section{3.- A MODO DE CONCLUSIÓN}

Sin perjuicio de las observaciones realizadas en las líneas precedentes, se presenta la siguiente tabla donde queda sintetizada nuestra propuesta para la virtualización de la enseñanza en Ciencias Jurídicas respecto de la asignatura "Teoría de las Relaciones Laborales" del Grado en Relaciones Laborales y Recursos Humanos por la Universidad de Granada. 


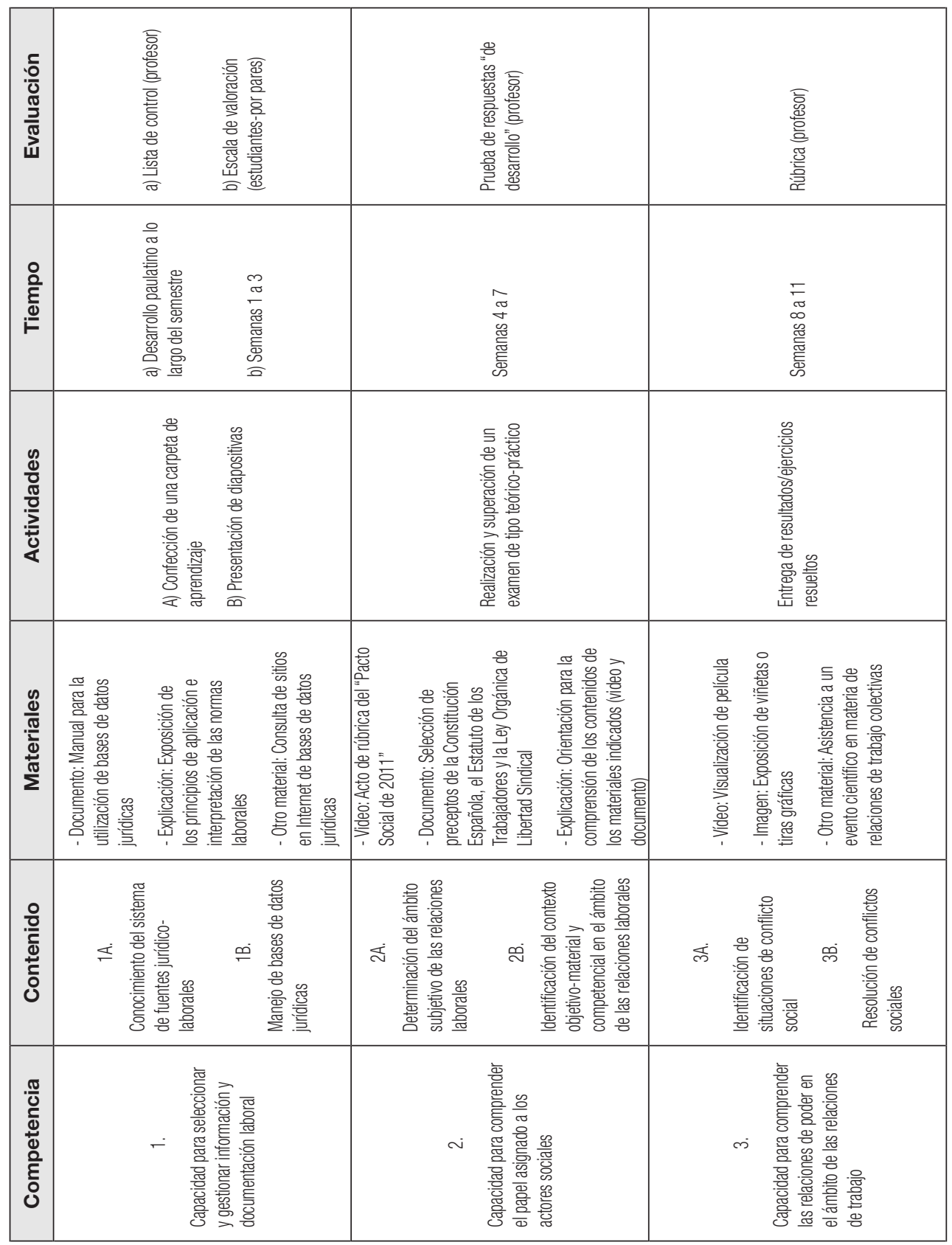




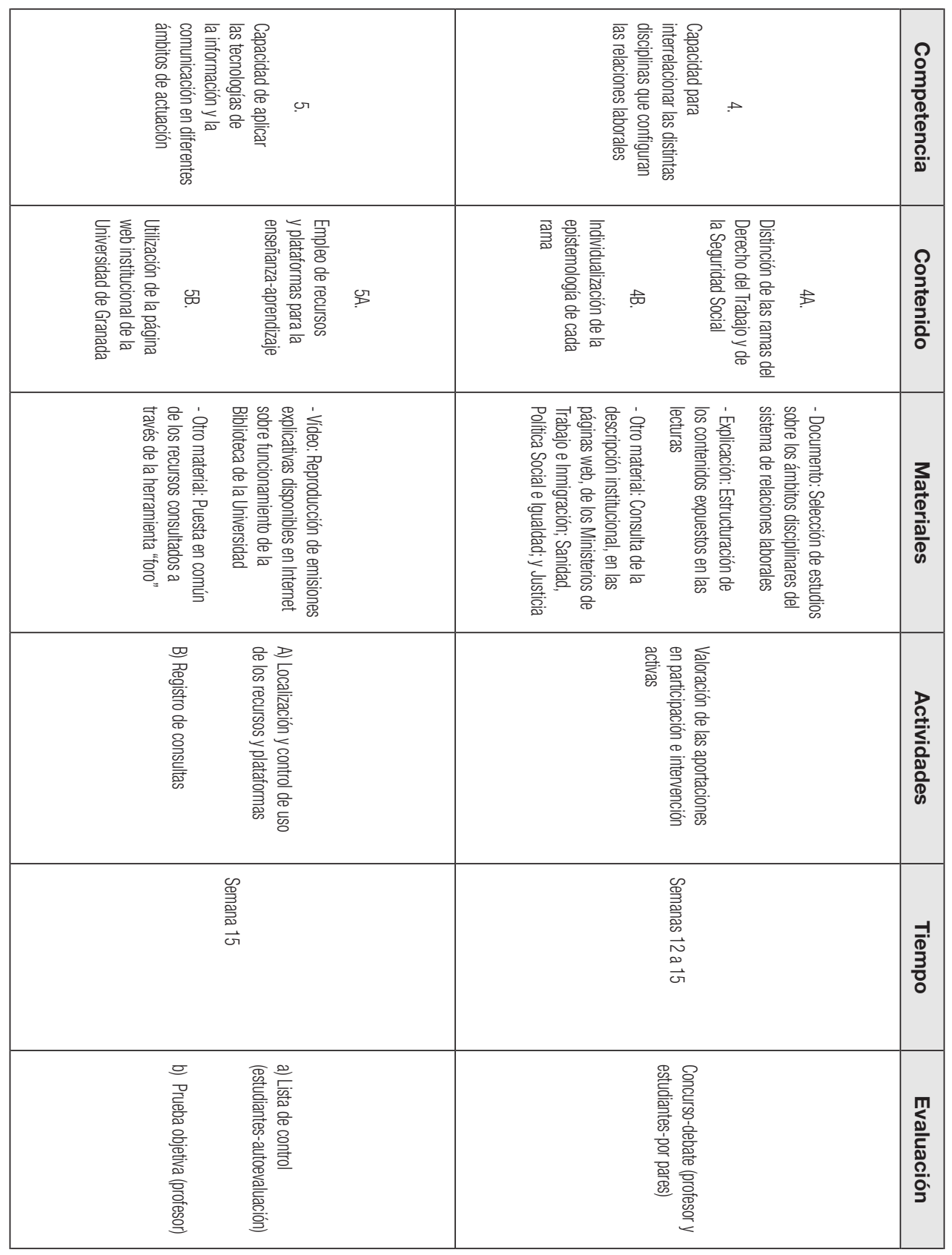




\section{4.- BIBLIOGRAFÍA}

Blanco Fernández, A. (Coord.) (2009): Desarrollo y evaluación de competencias en Educación Superior, Madrid, Narcea.

Cano García, M. A. (2008): "La evaluación por competencias en la Educación Superior", Profesorado: revista de curriculum y formación del profesorado, núm. 3.

Cebrián de la Senra, M. (2003): Enseñanza virtual para la innovación universitaria, Madrid, Narcea Ediciones.

Delgado García, A. M. (Coord.) (2006): Evaluación de las competencias en el Espacio Europeo de Educación Superior. Una experiencia desde el Derecho y la Ciencia Política, Barcelona, Bosch.

De Miguel Díaz, M. (Coord.) (2006): Metodologías de enseñanza y aprendizaje para el desarrollo de competencias. Orientaciones para el profesorado universitario ante el Espacio Europeo de Educación Superior, Madrid, Alianza.

Goñi Zabala, J. M. (2005): El Espacio Europeo de Educación Superior, un reto para la Universidad: competencias, tareas y evaluación, los ejes del curriculum universitario, Barcelona, Octaedro. 